Paper O-28

\title{
Radiative snowflake divertor studies in DIII-D
}

V.A. Soukhanovskii ${ }^{a, *}$, S.L. Allena, M.E. Fenstermacher ${ }^{a}$, D.N. Hill ${ }^{a}$, C.J. Lasnier $^{a}$, M.A. Makowski ${ }^{a}$, A.G. McLean ${ }^{a}$, W.H. Meyer ${ }^{a}$, E. Kolemen ${ }^{b}$, R.J.

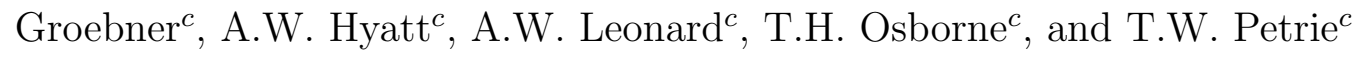

${ }^{a}$ Lawrence Livermore National Laboratory, P.O. Box 808, Livermore, CA 94550, USA

${ }^{b}$ Princeton Plasma Physics Laboratory, P.O. Box 451, Princeton, NJ 08543-0451, USA

${ }^{c}$ General Atomics, P.O. Box 85608, San Diego, CA 92186-5608, USA

\begin{abstract}
Recent DIII-D experiments assessed the snowflake divertor (SF) configuration in a radiative regime in $\mathrm{H}$-mode discharges with $\mathrm{D}_{2}$ seeding. The $\mathrm{SF}$ configuration was maintained for many energy confinement times $(2-3 \mathrm{~s})$ in H-mode discharges $\left(I_{p}=1.2 \mathrm{MA}, P_{N B I}=4-5 \mathrm{MW}\right.$, and $B \times \nabla B$ down (favorable direction toward the divertor)), and found to be compatible with high performance operation (H98y2 $\geq 1$ ). The two studied SF configurations, the SF-plus and the SF-minus, have a small finite distance between the primary X-point and the secondary $B_{p}$ null located in the private flux region or the common flux region, respectively. In H-mode discharges with the SF configurations (cf. H-mode discharges with the standard divertor with similar conditions) the stored energy lost per the edge localized mode (ELM) was reduced, and significant divertor heat flux reduction between and during ELMs was observed over a range of collisionalities, from lower density conditions toward a higher density H-modes with the radiative SF divertor.
\end{abstract}


Presenting and corresponding author Address: Lawrence Livermore National Laboratory, P.O. Box 808, Mailstop L-637, Livermore, CA 94551, USA. Email: vlad@llnl.gov

JNM keywords: P0500 Plasma-Materials Interaction, P0600 Plasma Properties

PSI-20 keywords: Divertor, Divertor plasma, Power deposition, ELM, DIII-D PACS Nos.: 52.55.Fa Tokamaks, 52.55.Rk Power exhaust, divertors 


\section{Introduction}

A snowflake (SF) divertor configuration [1] has been proposed as a potential solution for the tokamak divertor power exhaust problem. The SF divertor magnetic configuration uses a second-order null created by merging two first-order nulls of the standard divertor $[1,2]$. Poloidal magnetic flux surfaces in the region of the exact second-order null have six hexagonal separatrix branches with an appearance of a snowflake. The exact second-order null configuration is topologically unstable [1]. In the experiment, two variants of the exact configuration called snowflake-plus and snowflake-minus are often realized in steady-state. In the SF-plus, the secondary null is on the private flux region side of the standard divertor X-point. In the SF-minus, the secondary null approaches the standard divertor X-point from the common flux scrapeoff layer (SOL) side. In the SF configurations, the region of low poloidal field $B_{p}$ surrounding the null(s) (Fig. 1) is broader (cf. standard divertor) and has a strong impact on edge plasma properties. Experiments performed in the TCV $[3,4,5]$, NSTX $[6,7,8]$ and DIII-D tokamaks [9] are providing data to support the physics basis for the SF divertor concept development for future high-power facilities. Initial experiments in DIII-D and NSTX at high divertor power density demonstrated significantly reduced divertor heat flux with the SF-minus divertor and compatibility with high performance operation (H98y2 $\geq 1$ ). In this paper we discuss recent DIII-D experiments where the $\mathrm{SF}$ configurations were combined with $\mathrm{D}_{2}$-seeded $\mathrm{H}$-mode plasmas to assess 
the SF geometry effects, heat transport, and radiation in the radiative SFplus and SF-minus divertors. Reduced ELM energies, and greatly reduced divertor inter-edge localized mode (ELM) and peak ELM heat fluxes were measured in the SF-minus and SF-plus divertor configurations spanning a range of edge and divertor collisionalities (e.g. with attached and partially detached strike points), compatible with high performance H-mode operation.

\section{Experimental}

The SF divertor configuration experiments were conducted in DIII-D using a standard highly-shaped H-mode discharge scenario with $I_{p}=1.2 \mathrm{MA}$ and $P_{N B I}=1-5 \mathrm{MW}$, and ion $B \times \nabla B$ direction toward the lower divertor. A divertor cryo-pump was used for particle removal, and $\mathrm{D}_{2}$ seeding was used for steady-state density control in the range $(0.4-0.7) \times n_{e} / n_{G}$ $\left(\sim 4.5-7.5 \times 10^{19} \mathrm{~m}^{-3}\right.$ where $n_{G}$ is the Greenwald density [10]). The DIII-D tokamak divertor is an open geometry divertor with graphite plasma-facing components and divertor heat fluxes of several $\mathrm{MW} / \mathrm{m}^{2}$.

Snowflake configurations were obtained using three existing poloidal field shaping coils in the divertor region. The SF-minus configuration used a combination of a pre-programmed coil current and strike point position control by the plasma control system (as in Ref. [7]), while the SF-plus was explicitly controlled through a recently developed algorithm [11]. Both configurations were produced for long periods exceeding energy confinement time $\tau_{E} \leq 0.250 \mathrm{~s}$ and comparable with discharge duration of $5-6 \mathrm{~s}$. Examples of the standard divertor, SF-plus and SF-minus magnetic equilibria with 
their additional strike points (SP) are shown in Fig. 1. Magnetic equilibria were reconstructed using the standard Grad-Shafranov based equilibrium code EFIT. Magnetic field structure and geometric properties of the SF-plus and the SF-minus are similar to those of the exact SF configuration when the distance $D$ between the poloidal nulls satisfies $D \leq a\left(\lambda_{q} / a\right)^{1 / 3}$, where $a$ is the minor radius and $\lambda_{q}$ is the SOL power width as projected to midplane [2]. The criterion yields $D \leq 10 \mathrm{~cm}$ for the DIII-D parameters $a$ and $\lambda_{q}$, while in the experiment the distance $D \leq 10 \mathrm{~cm}$ was typically realized, so it was expected that the SF-plus and the SF-minus would behave much like the exact SF. If the divertor poloidal null positions in normalized poloidal flux $\psi_{N}$ in the SF-minus configuration are remapped to the mid-plane, as is commonly done for the $d r_{\text {sep }}$ parameter in double-null magnetic configurations, one obtains $d_{X X} \leq 1-2 \mathrm{~mm}$ (cf. SOL power width $\lambda_{q}=2.5-3 \mathrm{~mm}$ $[12])$.

\section{Results}

\subsection{Impact of magnetic geometry on divertor inter-ELM heat fluxes}

Divertor magnetic geometry modifications in the SF configuration are predicted to affect divertor heat and particle transport and power deposition in a favorable way $[1,2]$. In this work we focus on the outer SOL and divertor, as the inner strike point on the vertical target received small amounts of heat flux in both SF and standard configurations: $q_{i n} \leq 0.1-0.3 \mathrm{MW} / \mathrm{m}^{-3}$ between ELMs. The SF properties realized in the DIII-D experiments included: formation of the additional strike points, increased connection length $L_{\|}$, increased plasma-wetted area $A_{\text {wet }}=2 \pi R_{S P} f_{\text {exp }} \lambda_{q}$ (where $f_{\text {exp }}$ is the poloidal 
magnetic flux expansion) via $f_{\text {exp }}$, and increased specific divertor volume (proportional to $L_{\|}$). These points are illustrated in Fig. 1 where the typical standard and SF divertor configurations, the extent of the low $B_{p}$ region, and the $f_{\text {exp }}$ and $L_{\|}$radial profiles are compared. In the SF-plus and SF-minus configurations, the zone of low $B_{p}$ was broad and encompassed additional divertor legs and strike points, potentially leading to a much larger region with high poloidal beta, where fast convective plasma redistribution driven by toroidal curvature-driven flute-like modes [2] could take place. In the SF-plus, the secondary $B_{p}$ null (in the private flux region) mainly affected the geometry at the separatrix, within $\leq 10 \%-20 \%$ of the SOL width of $3 \mathrm{~mm}$ (in 1.2 MA discharges). The plasma-wetted area increase due to the flux expansion and the connection length increase were modest (10\%-30\%). In the SF-minus, the secondary null separated the outer SOL into two manifolds. In the manifold formed between the primary and secondary separatrices (nulls), heat flow was significantly affected by the geometry as both the $f_{\text {exp }}$ and $L_{\|}$ were increased by up to $70 \%$. In the second, outside SOL manifold, the geometry was modified to a lesser extent.

These magnetic geometry modifications led to heat flux reduction and power spreading in the divertor (cf. standard divertor). Shown in Fig. 2 are the outer divertor heat flux profiles measured in H-mode discharges with $P_{N B I}=4 \mathrm{MW}$ and at low $n_{e} \sim(5-6) \times 10^{19} \mathrm{~m}^{-3}$. The inter-ELM profiles are conditionally averaged during the last $25 \%$ of the inter-ELM cycle, i.e. before the ELM when the heat flux is fully relaxed. The profiles are sampled within 100-200 ms between 4 and $5 \mathrm{~s}$ of each discharge, at $n_{e}$ matched between the standard and the SF discharges. In the SF-minus, heat flux 
in the main separatrix strike point SP1 was measured only at much higher input power (e.g., at $P_{i n} \sim 11 \mathrm{MW}$ [13]), or during ELM transients. The peak heat flux reduction in the additional outer strike point SP3 was to a large extent dominated by the $f_{\exp }$ factor, and varied within $50 \%$, as shown in Fig. 2(a), for seemingly identical discharge conditions. In the SF-plus, the $f_{\exp }$ impact on the heat deposition in the primary separatrix strike point SP1 was minimal, suggesting that other geometry (aside from the $f_{\text {exp }}$ ) and/or transport effects contributed to the heat flux reduction. Divertor radiation in these discharges was low and similar within 10-15\% between the SF and standard divertor, hence not a significant factor in the power balance. Radial spreading due to enhanced SOL transport and/or longer $L_{\|}$in the SF could lead to greater $\lambda_{q}$ and hence contribute to the increased $A_{\text {wet }}$. The analysis will be presented elsewhere. The inner (vertical) target heat fluxes were affected in a similar manner.

\subsection{Radiative SF divertor}

The SF geometry could lead to a facilitated access to the strike points detachment due to increased volumetric power and momentum losses, as apparently was the case in the NSTX SF-minus experiments [7]. Radiative divertor conditions in DIII-D are routinely accessed with carbon and deuterium radiation using $\mathrm{D}_{2}$ seeding that increases upstream (and core) density $[14,15]$.

Initial radiative $\mathrm{SF}$ divertor experiments demonstrated that: 1) both the radiative SF-plus and SF-minus were compatible with the H-mode albeit with some confinement degradation; 2) the reduction of inter-ELM divertor heat fluxes was stronger in the SF configurations; 3) carbon and deuterium 
emissions were more broadly distributed in the SF configurations, including the additional divertor legs; 4) the onset of radiative SF conditions (e.g., increase in impurity radiation and recombination, heat flux reduction) were obtained at similar core $n_{e}$ as in comparable discharges with the standard divertor.

While the confinement degradation was not associated with the SF formation at lower-to-medium densities, additional $\mathrm{D}_{2}$ seeding at rates $50-80$ Torr $\mathrm{l} / \mathrm{s}$ (to raise the density for radiative divertor onset) resulted in 10\%-20\% reduction in, e.g., H98(y,2) and H89L factors and plasma stored energy $W_{M H D}$ with the standard radiative divertor, and up to 20-40\% in H-mode discharges with the radiative (higher-density) SF-plus or SF-minus. The degradation was associated with the reduction of pedestal $T_{e}^{p e d}$ and pedestal energy $W_{\text {ped }}=3 / 2 n_{e}^{\text {ped }}\left(T_{e}^{\text {ped }}+T_{i}^{\text {ped }}\right) V_{\text {plasma }} \simeq 3 P_{e}^{\text {ped }} V_{\text {plasma }}$. Further H-mode scenario development is necessary to optimize compatibility of the core plasma with radiative $\mathrm{SF}$, as is typically done with the standard radiative divertor (e.g., Ref. [16]).

Inter-ELM divertor heat flux profiles demonstrated that the radiative SF divertor was more effective in reducing divertor peak heat loads than the standard divertor at $P_{N B I}=4-5 \mathrm{MW}$. The profiles are compared in Fig. 3. In the standard divertor, the partial detachment led to a significant (up to x10) peak heat flux reduction. In the radiative SF-minus and SF-plus, the reduction was greater, leading to a nearly complete power detachment, as heat flux in the outermost strike point was barely measurable. The $f_{\exp }$ factor accounted for most of the difference between the radiative SF-minus and the standard divertor, while being insufficient in the SF-plus case (similar 
to the attached SF case discussed in Sec. 3.1).

Divertor radiated power (from carbon and deuterium species) was distributed more broadly and uniformly in the SF configurations. Shown in Fig. 3(c,d) are radiated power distributions in the standard and SF-minus divertors as they progress toward highly radiative conditions, obtained from tomographic reconstructions of divertor radiated power measured by multichannel bolometry. The total divertor radiated power was about $2 \mathrm{MW}$ in the radiative standard, SF-plus or SF-minus configurations, differing by $10 \%$ $15 \%$ (cf. $P_{S O L} \sim 3.0-3.5 \mathrm{MW}$ ). In the standard divertor, radiation initially peaked in the inner and outer divertor legs, and eventually the radiative front moved to the X-point (e.g., Ref. [15]). In the SF-minus, radiation also initially peaked in the divertor legs, however, as the SF-minus was formed, it was broadly distributed throughout the divertor volume, with occasional peaking at the null-points. In the SF-plus, the radiation front was formed in the divertor legs and moved toward the null-point region where it stabilized. The extended connection length region enabled a broader radiation zone. Importantly, despite the geometry (e.g., increase in $L_{\|}$by $50 \%-75 \%$ ), the SF configurations were not more likely than the standard divertor to form $\mathrm{X}$-point radiative instabilities that can degrade the confinement.

\subsection{ELM heat flux mitigation}

The SF configuration effects on pedestal and SOL led to the reduction of ELM energy and heat deposition on the targets at lower density, and a near complete elimination of ELM heat fluxes in highly radiative conditions. Previously, similar observations were made in TCV [5] and NSTX [8]. In the DIII-D H-mode discharges, the majority of ELMs were Type I ELMs with 
$\Delta W_{E L M} / W_{p e d} \sim 0.02-0.1$, with a few larger ELMs.

In the pedestal region, both the magnetic shear and $q_{95}$ were systematically increased by $10 \%-30 \%$ due to the broader region of lower $B_{p}$ inside the separatrix. Accordingly, the pedestal stored energy lost per ELM $\Delta W_{E L M}$ was reduced since the pedestal collisionality $\nu_{p e d}^{*}=\pi R q_{95} / \lambda_{e, e}$ was increased and the ELM parallel transit time $\tau_{\|}^{E L M} \sim L_{\|} / c_{s, p e d}$ (the pedestal ion transport time from the mid plane to the target at the sound speed $c_{s}$ ) was increased. This was consistent with the Type I ELM scaling of $\Delta W_{E L M}$ with $\nu_{\text {ped }}^{*}$ found in many tokamaks [17]. Shown in Fig. 4 are pedestal and ELM characteristics in the standard and the SF-minus divertor discharges at lower densities and at radiative conditions. Kinetic profiles were similar with and without the SF configurations. Pedestal top plasma parameters changed within 5\%-15\%: with the SF configuration, $T_{e}^{\text {ped }}$ slightly reduced, $n_{e}^{\text {ped }}$ slightly increased, and $p_{e}^{p e d}$ remained nearly constant, as shown in Fig. 4(a). Pedestal energy $W_{\text {ped }}$ was nearly unaffected at lower $n_{e}$. However, some additional degradation of the pedestal $T_{e}$ was noted in highly radiative SF configurations, leading to the pedestal energy reduction [Fig. 4(a,b)]. The pedestal MHD stability was apparently weakly affected by the magnetic shear and pedestal pressure gradient changes: only a small increase in ELM frequency $f_{E L M} \leq 20 \%$ was detected with the SF. The stored energy lost per ELM $\Delta W_{E L M}$ was reduced in discharges with the SF configurations. In some discharges, the effect was strong, $\Delta W_{E L M}$ was reduced by up to $50 \%$ [9]. More typically, however, the reduction was in the range 5\%-20\%. At higher density in radiative SF divertor discharges, both the $\Delta W_{E L M}$ and $\Delta W_{E L M} / W_{p e d}$ were lower by 10\%-20\% [cf. standard divertor, Fig. 4(b,c)]. 
The SF configurations also produced lower peak target temperatures and reduced heat loads (cf. standard divertor) due to ELMs. The increased divertor connection length $L_{\|}$can reduce the target surface temperature rise due to pulsed heat load during an ELM as $\Delta T_{t} \sim W_{E L M} / \sqrt{\tau_{d}}$, where $W_{E L M}$ is the ELM energy and $\tau_{d}$ is the ELM deposition time which is increased at longer $L_{\|}[18]$. Another possible mechanism is the fast convective transport in the low $B_{p}$ region driven by instabilities [2] that can lead to the ELM heat flux sharing among the additional strike points. Shown in Fig. 4(d) is the divertor power operating space, the total power received by the outer target $P_{d i v}^{o u t}$ vs the power received by the inner target $P_{d i v}^{i n}$, for the discharges discussed above. The total power is obtained by integrating heat flux profiles measured by infrared thermography. Outer peak powers above 1-2 MW are attributed to ELMs in the standard and SF-minus at lower $n_{e}$. The peak powers were reduced in the SF-minus by up to $50 \%-70 \%$, and further reduced in the radiative SF-minus by up to $50 \%$, as compared to the standard divertor configurations. Some uncertainties remain as to whether the SF configurations were maintained during equilibria perturbations due to large ELMs. Divertor power deposition measurements (i.e., strike point dynamics) from fast infrared thermography suggested that in most cases the SF configurations were not destroyed. In the standard divertor configuration, radiative buffering of ELM divertor heat loads has not been effective (e.g., Ref. [19]). Typically, the partially-detached standard divertor strike points re-attach during ELMs and significant transient heat and particle fluxes reach the targets. Radiative SF divertor experiments in DIII-D demonstrated that at increased density (collisionality), both the $\Delta W_{E L M}$ and the divertor $q_{p e a k}^{E L M}$ 
were reduced stronger than in standard radiative divertor, leading to the much reduced peak powers. A combination of the geometry, transport and enhanced radiative dissipation may provide a significant benefit for ELM buffering. We note that this observation was also made in NSTX radiative SF-minus discharges [8].

In summary, the emerging understanding of inter-ELM and ELM divertor heat transport in the radiative SF divertor from recent DIII-D experiments provides support to the snowflake divertor concept as a promising solution for divertor heat flux mitigation in future magnetic fusion devices. The experiments demonstrated the SF divertor compatibility with high H-mode confinement, radiative divertor with gas seeding, and led to significantly reduced ELM energies, as well as divertor heat fluxes between and during ELMs. Future experiments and analysis will focus on confinement optimization, transport, radiative limit, and cryo-pumping compatibility studies, as well as magnetic feedback control improvements.

\section{Acknowledgments}

This work was performed under the auspices of the U.S. Department of Energy (US DOE) under DE-AC52-07NA27344, DE-AC02-09CH11466, and DE-FC02-04ER54698. DIII-D data shown in this paper can be obtained in digital format by following the links at https://fusion.gat.com/global/D3D_DMP. We thank the entire DIII-D Team for technical, engineering and computer support as well as plasma and diagnostic operations. Dr. D.D. Ryutov is acknowledged for insightful discussions. 


\section{References}

[1] D. Ruyutov, Phys. Plasmas 14 (2007) 064502.

[2] D. Ryutov et al, Plasma Phys. Control. Fusion 54 (2012) 124050

[3] F. Piras et al., Phys. Rev. Lett. 105 (2010) 155003.

[4] H. Reimerdes et al., Plasma Phys. Control. Fusion 55 (2013) 124027

[5] W. A. Vijvers et al., Nucl. Fusion 54 (2014) 023009.

[6] V.A. Soukhanovskii et al., Nucl. Fusion 51 (2011) 012001.

[7] V.A. Soukhanovskii et al., Phys. Plasmas 19 (2012) 082504.

[8] V.A. Soukhanovskii et al., J. Nucl. Mater. 438 (2013) S96.

[9] D.N. Hill Nucl. Fusion 53 (2013) 104001.

[10] M. Greenwald et al., Nucl. Fusion 28 (1988) 2199.

[11] E. Kolemen, this conference, Paper O-18 (2014).

[12] M.A. Makowski et al., Phys. Plasmas 19 (2012) 056122.

[13] T.W. Petrie, this conference Paper P1-082 (2014).

[14] T.W. Petrie et al., Nucl. Fusion 37 (1997) 321.

[15] M.E. Fenstermacher et al., Plasma Phys. Control. Fusion 41 (1999) A345.

[16] T.W. Petrie et al., J. Nucl. Mater.-363-365 (2007) 416. 
[17] A. Loarte et al., J. Nucl. Mater. 313-316 (2003) 962.

[18] T. Rognlien et al., J. Nucl. Mater. 438 (2013) S418.

[19] P. Monier-Garbet, Nucl. Fusion 45 (2005) 1404. 
Figure 1: Equilibria of the standard, SF-minus, and SF-plus divertor configurations (a). The primary separatrix is shown in red, the secondary in blue. The region of low $B_{p} \leq$ $0.1\left|B_{p} / B_{p m}\right|$ is also shown. (b) Magnetic flux expansion $f_{\text {exp }}$ profile vs divertor distance from strike point SP1. (c) Midplane-to-target connection length $L_{\|}$(c) profile vs radial distance from separatrix remapped to the outer midplane radius (cf. $\lambda_{q}$ ).

Figure 2: Inter-ELM divertor heat flux profiles in the standard, SF-minus (a) and SF-plus (b) configurations in $P_{N B I}=4 \mathrm{MW} \mathrm{H}$-mode discharges at lower $n_{e}$.

Figure 3: Inter-ELM divertor heat flux profiles in the standard radiative, SF-minus (a), and SF-plus (b) divertors. Radiated power density distribution during a transition to radiative divertor in the standard (c) and SF-minus (d).

Figure 4: Pedestal and divertor parameters in H-mode discharges with the standard and SF-minus configurations at lower $n_{e}$, and the radiative standard and SF-minus divertors at higher $n_{e}$. Data sampled between 4 and $5 \mathrm{~s}$. (a) Pedestal operating space, $T_{e}^{\text {ped }}$ vs $n_{e}^{\text {ped }}$; (b) Stored energy lost per ELM vs pedestal energy; (c) Normalized energy lost per ELM $\Delta W_{E L M} / W_{\text {ped }}$ vs pedestal Greenwald density fraction; (d) Divertor power operating space, $Q_{\text {out }}(t)$ vs $Q_{i n}(t)$. 
Figure 2

2 Divertor heat flux $\left(\mathrm{MW} / \mathrm{m}^{2}\right)$

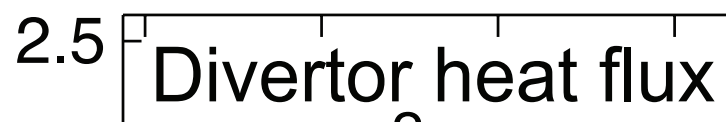

Standard

SF-minus

SF-minus

0

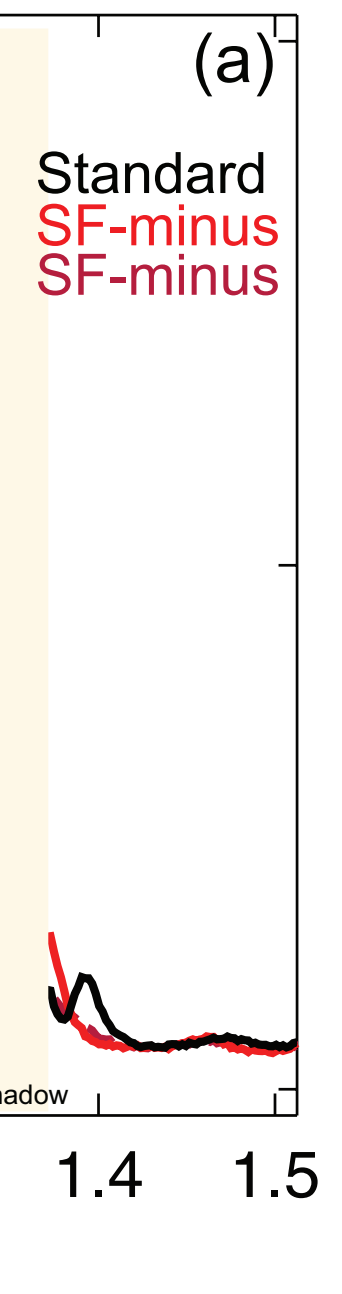

1

\section{0}
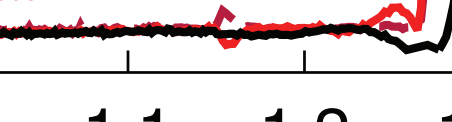

$R \operatorname{div}(\mathrm{m})$

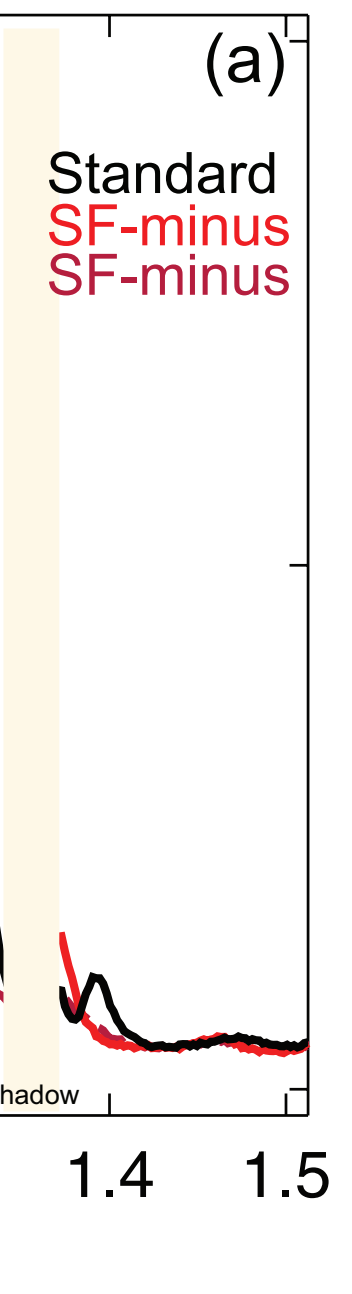

\section{-}

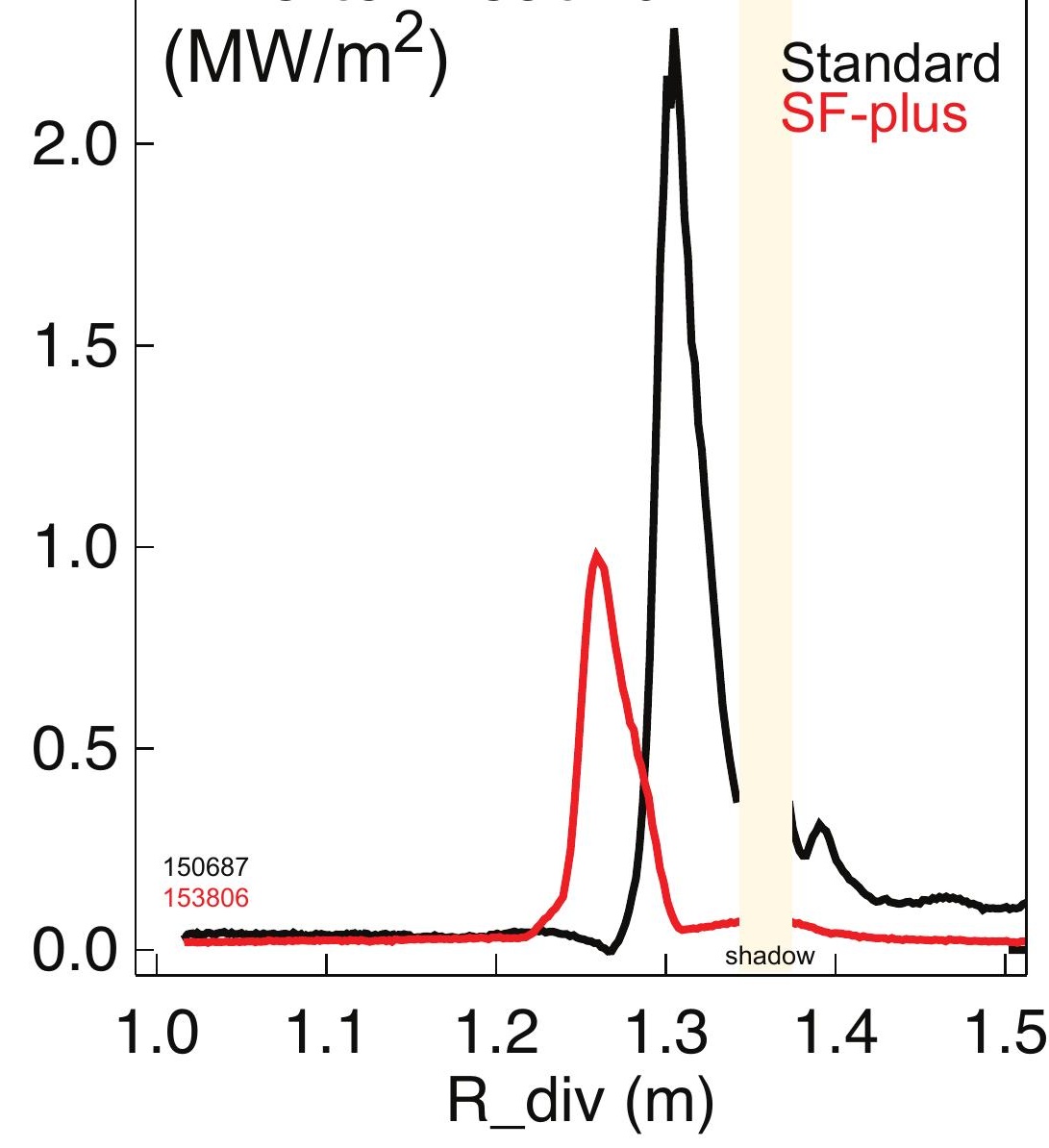

(b)

Standard SF-plus

\section{R_div (m)}



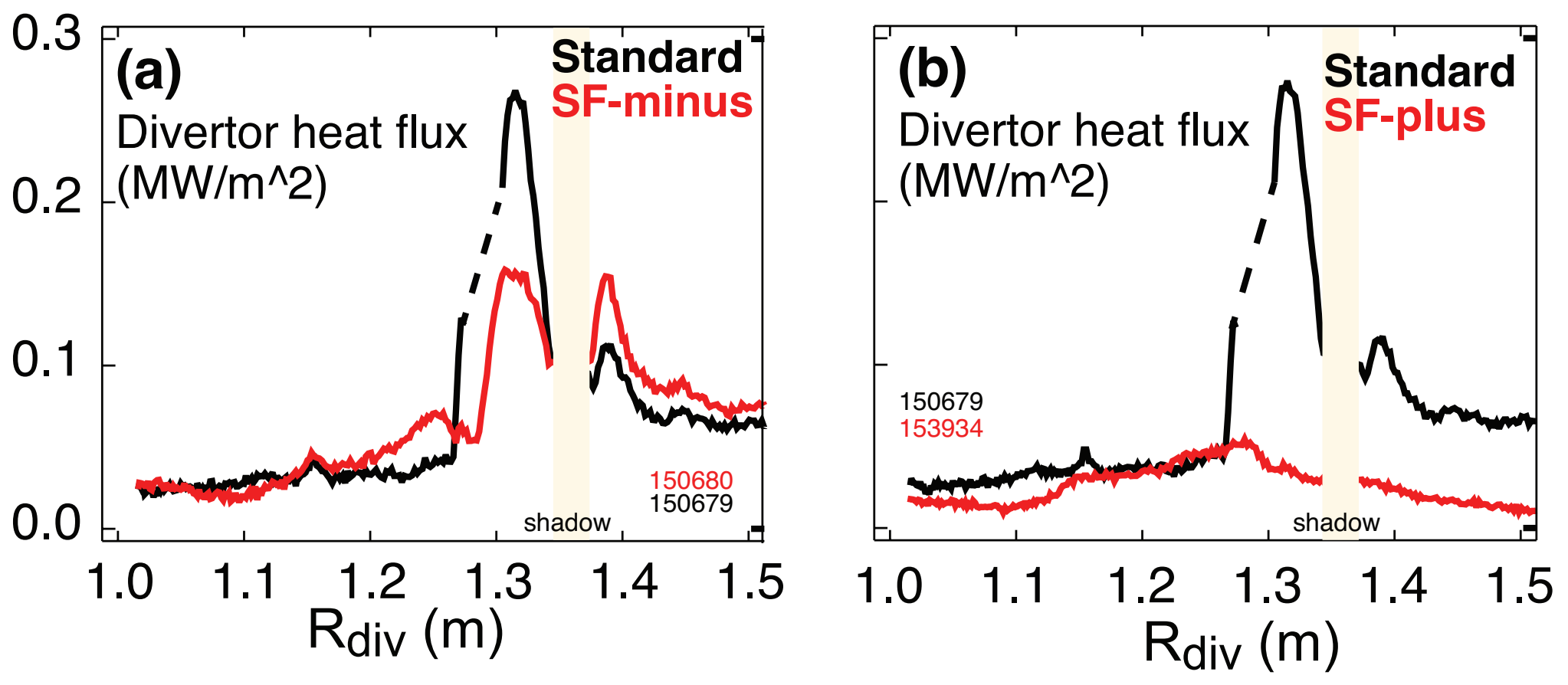

\section{Standard radiative divertor}

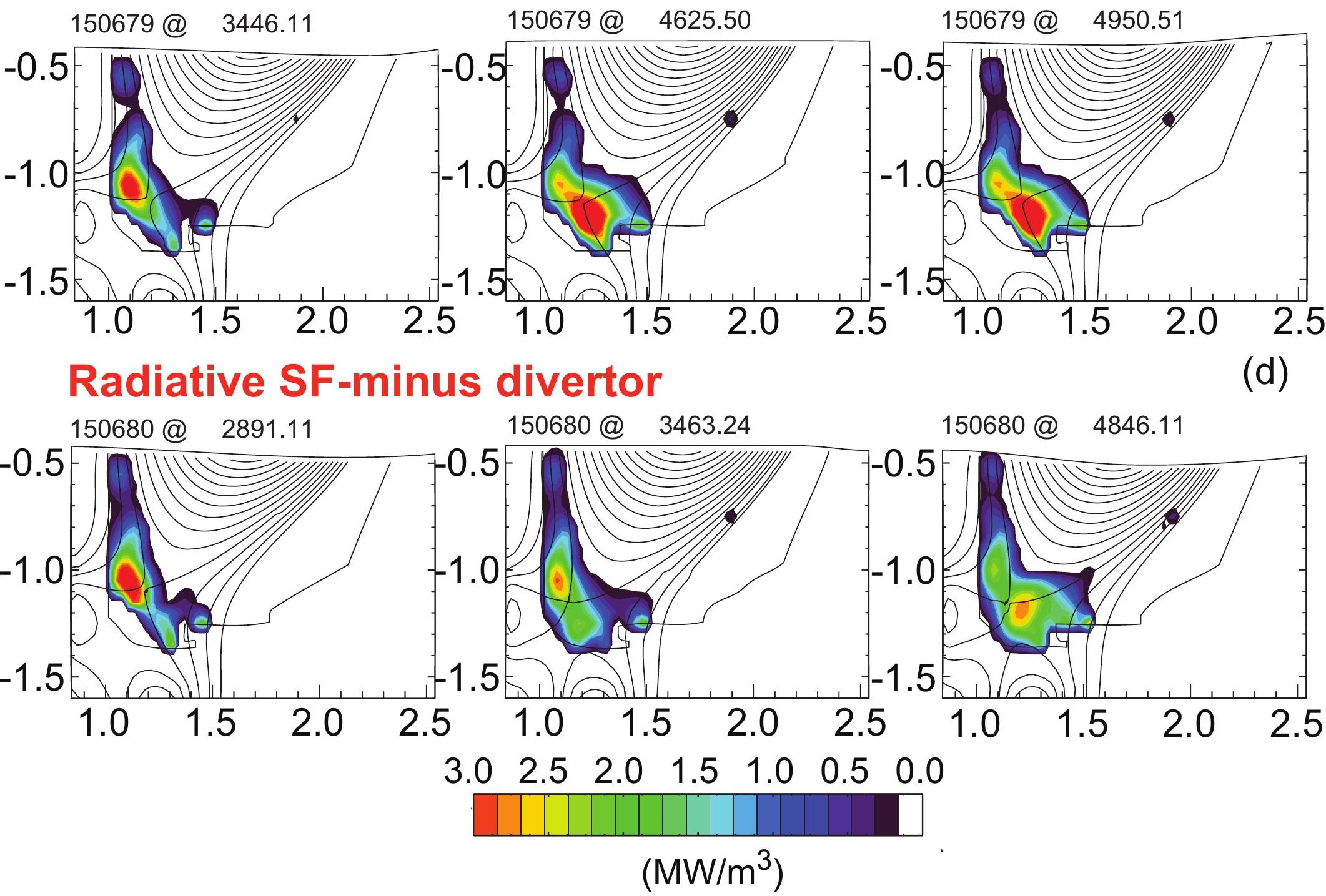



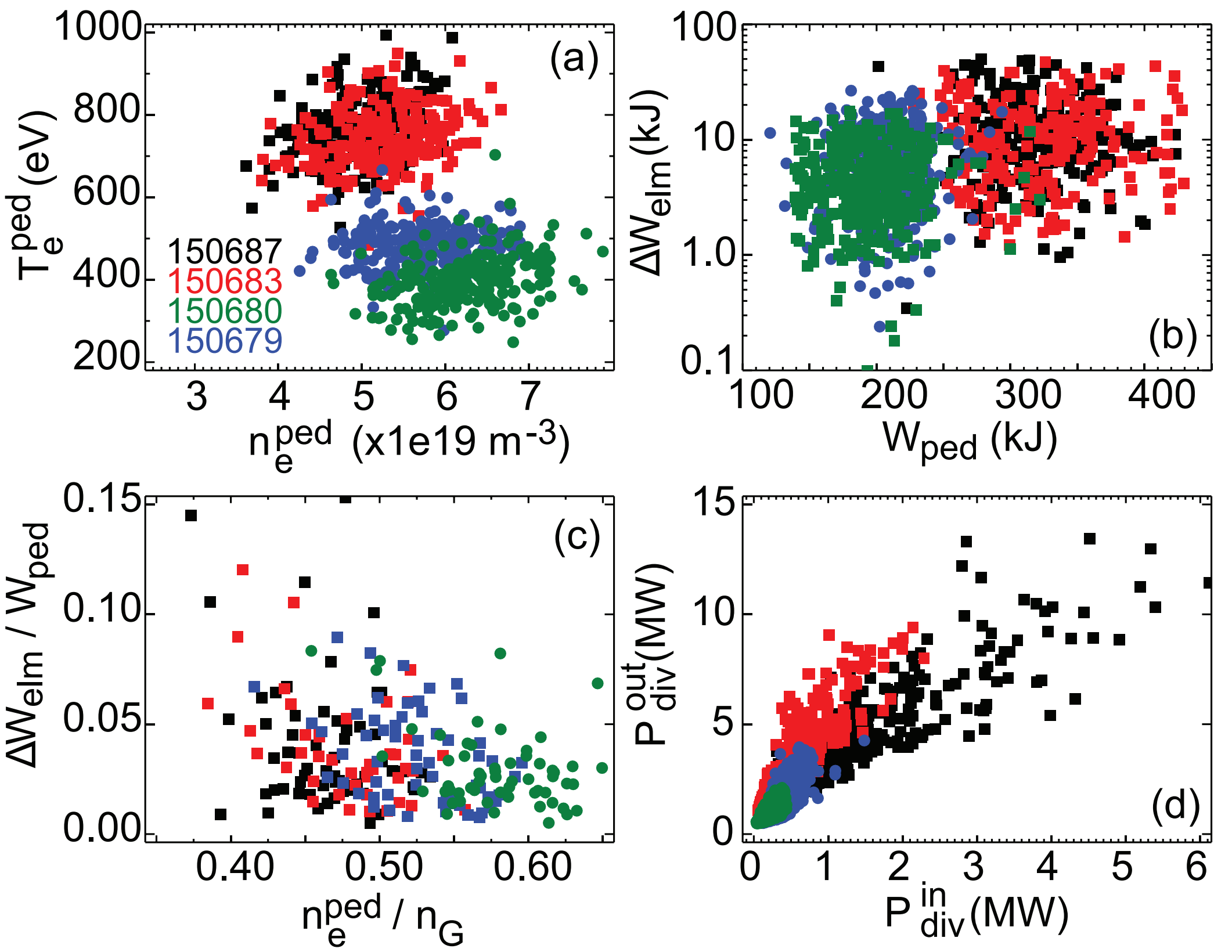

Color coding:

Standard SF-minus Radiative Standard Radiative SF-minus 